\title{
El rol de la perspectiva de tiempo futuro en la motivación estudiantil ${ }^{1}$
}

\author{
Willy Lens \\ Universidad Católica de Lovaina \\ Lovaina, Bélgica
}

El propósito de este artículo es explicar cómo la motivación de los estudiantes para el aprendizaje y las tareas de logro derivan parcialmente de su utilidad o instrumentalidad para el cumplimiento de metas en el futuro cercano o distante. La pregunta central es ¿cómo la orientación hacia el futuro o la perspectiva de tiempo futuro (PTF) de los estudiantes afecta su motivación y su orientación hacia la meta? Se presenta el modelo teórico que explica los efectos motivacionales de las diferencias individuales en la PTF y luego se aplica el modelo a la motivación estudiantil. Después de revisar trabajos sobre la interacción entre la motivación intrínseca y las recompensas extrínsecas, el autor discute los efectos motivacionales de la instrumentalidad o utilidad de dicha interacción para los diferentes tipos de metas futuras.

motivación intrínseca / motivación extrínseca / distancia psicológica / instrumentalidad

\section{The role of future time perspective in student motivation}

The purpose of the article is to explain how student motivation for learning and achievement tasks partly derive from their utility or instrumentality for goals in the near or distant future: how does the future time perspective (FTP) of students affect their present motivation and goal orientation? FTP as a motivational variable in discussed. A theoretical model that explains the motivational effects of individual differences in FTP is introduced and later applied to student motivation. After reviewing recent research on the interaction between extrinsic rewards and intrinsic motivation, the author discusses the motivational effects of utility or instrumentality for different kinds of future goals.

\section{intrinsic motivation / extrinsic motivation / psychological distance / instrumentality}

1 Traducción del inglés por Ricardo Braun. Revisión técnica: Dora Herrera. 
El aprendizaje y los logros en la escuela son actividades intencionales orientadas hacia determinadas metas. Los estudiantes motivados pueden tener varias y muy diferentes razones para estudiar: para desarrollar sus habilidades cognitivas; para saber más y ser capaz de resolver problemas complejos y difíciles; porque disfrutan el trabajo con sus pares; porque quieren complacer a sus maestros o padres, de quienes esperan recibir alguna recompensa; o quieren ser exitosos y no fracasar. Algunas veces están motivados para superarse a sí mismos, a sus hermanos o amigos. Muchos estudiantes en escuelas primarias y secundarias están positivamente motivados en sus estudios porque quieren entrar a la universidad o porque quieren tener una profesión o empleo específico cuando sean adultos.

Las metas individuales que se buscan a través del aprendizaje y logros en la escuela pueden ser ubicadas en dos dimensiones: metas intrínsecas versus extrínsecas y metas inmediatas versus futuras. Algunas de estas metas pueden situarse en un futuro inmediato o muy cercano; otras pueden estar ubicadas en el futuro lejano y sólo pueden ser obtenidas si el estudiante ha logrado completar una serie de submetas (calificaciones en la secundaria, universidad, etc.) una después de la otra.

La motivación total para aprender es la combinación de motivaciones intrínsecas y extrínsecas. Se dice que los niños están intrínsecamente motivados cuando el aprendizaje y desempeño en la escuela constituyen una meta en sí misma. En cambio, están extrínsecamente motivados cuando la actividad se realiza en razón al material mismo y a otras recompensas que no están intrínsecamente relacionadas con el aprendizaje escolar. Así, aprender y tener buenos resultados en los exámenes se constituyen en actividades instrumentales para lograr dichas recompensas. Una estudiante puede estar muy motivada para un curso de matemáticas por diversas razones. Puede haber estado intrínsecamente interesada en los números y operaciones matemáticas desde niña y como es buena en números y los disfruta, realmente quiere convertirse en una experta en la materia. Trabaja fuerte para obtener una buena calificación en el examen final porque no sólo quiere ser promovida al siguiente grado sino porque sus padres le han prometido un equipo de música si obtiene buenos resultados. Tanto padres como maestros utilizan una serie de recompensas extrínsecas y otras medidas de control para incrementar la intensidad de la motivación para estudiar. Para los estudiantes sin una motivación intrínseca, éstas son las únicas razones para estudiar. Las investigaciones sobre motivación estudiantil se han concentrado fundamentalmente en los diferentes tipos de motivación intrínseca (Ames \& Ames, 1984; Malone \& Lepper, 1987; Pintrich \& Schunk, 1996) y en cómo la motivación intrín- 
seca es afectada por las recompensas y otras variables de control (Deci \& Ryan, 1985, 1992; Eisenberger \& Cameron, 1996).

Además de la lista de razones intrínsecas y extrínsecas, nuestra mencionada estudiante está altamente motivada para su curso de matemáticas porque ésta es una asignatura muy importante para ella. En efecto, pretende convertirse en una brillante ingeniera que le permita ganar mucho dinero para independizarse de sus padres o pareja, comprar autos de lujo y quizás jubilarse anticipadamente e ir a una isla griega. Se puede decir entonces, que tiene metas intrínsecas y extrínsecas cercanas o inmediatas, pero también varias metas extrínsecas y profesionales en el futuro mediano y lejano para las que la escuela tiene un valor instrumental o de utilidad.

El propósito de esta contribución es explicar cómo la motivación estudiantil para el aprendizaje y logros deriva de la utilidad o instrumentalidad de las metas en el futuro cercano o lejano. La pregunta es ¿cómo la orientación futura o perspectiva de tiempo futuro afecta la motivación presente y la orientación hacia las metas? Los padres y maestros suelen motivar a sus estudiantes refiriéndose a la utilidad e instrumentalidad de los estudios: “ $¡ E s-$ fuérzate en la escuela, es importante para tu futuro!" es un buen consejo que hemos recibido una y otra vez de nuestros padres, y muchos de nosotros lo empleamos hoy en día para motivar a nuestros hijos o estudiantes. Pero el hecho de que muchos jóvenes actualmente vivan con una perspectiva de tiempo futuro más bien corta, negativa y pesimista, crea un problema motivacional.

Perspectiva de TIEMPo Futuro: unA VARIABLE MOTIVACIONAL

\section{Determinación de la meta motivacional y la perspectiva de tiempo futuro}

La perspectiva de tiempo se refiere al pasado, presente y futuro cronológico en tanto ellos son parte del espacio de vida psicológica del individuo. La perspectiva de tiempo futuro (PTF) es, en consecuencia, el grado y la forma en que el futuro cronológico se integra en el espacio de vida del individuo (Lewin, 1942). La integración del futuro en el presente es análoga a la integración de lugares más o menos remotos en el espacio de vida actual. Ser consciente de lugares y distancias en el espacio y de momentos o intervalos de tiempo (pasado y futuro) es necesario pero no suficiente para otorgarles un gran efecto psicológico. La distancia psicológica de un lugar determinado puede diferir considerablemente entre las personas que viven en una misma ciudad. Para muchos europeos, París está psicológicamente muy lejos. No considerarían realizar una acción que los hiciera viajar hasta allá. Para otros, París está como en su patio. De manera 
análoga a la experiencia psicológica del mundo espacial, existen varias y profundas diferencias en la extensión o profundidad de la perspectiva de tiempo futuro. Algunas personas tienen una corta PTF. Sólo el futuro cronológico inmediato es parte del mundo temporal en que viven. Lo que venga después no lo toman en cuenta en el presente. Otras personas viven con una larga PTF. Su vida temporal presente se extiende hacia el futuro lejano. No tienen problemas para tomar en cuenta o estar motivados por eventos o resultados de las acciones en el futuro lejano.

Conceptualizamos la perspectiva de tiempo futuro como una característica adquirida de la personalidad que resulta de las metas motivacionales y de la demora de la gratificación (Nuttin \& Lens, 1985; Lens, 1986). A nivel superficial, los seres humanos elaboran cognitivamente y concretizan sus necesidades, motivos y deseos en metas motivacionales específicas, estructuras medios-fin u otros planes y proyectos motivacionales. Los estudiantes, por ejemplo, no tienen realmente una necesidad de logro o una necesidad de autorrealización, más bien, planifican tener éxito en sus exámenes, entrar a la universidad, convertirse en maestros, agrónomos, etc. Es sólo después de tales especificaciones que las necesidades y motivos pueden afectar el comportamiento exterior. Tales metas motivacionales y planes conductuales pueden ser caracterizados por su contenido o dominio motivacional al cual pertenecen (v.g., objetos de alimentación, afiliación, curiosidad, éxito, poder) y por su localización espacio-temporal. Por definición, las metas motivacionales anticipadamente positivas o negativas están situadas en el futuro. Pero la distancia temporal a las submetas y metas finales puede variar desde muy corta (v.g., ir a nadar esta tarde) hasta muy larga (v.g., el estudiante que se prepara para el examen de ingreso a la universidad en donde intenta hacer una carrera de cirujano). Esta distancia puede extenderse aún más allá del tiempo de vida individual (v.g., ahorrar para el propio funeral; para ir al cielo). El formular metas motivacionales distantes y el desarrollar proyectos conductuales de largo plazo para alcanzar dichas metas crea una perspectiva de tiempo futuro larga o extendida. Como tal, la PTF puede ser definida como la anticipación presente de las metas futuras. Algunas personas viven con una PTF más bien corta. Muchas de sus metas realistas están ubicadas en el futuro cercano. En el caso de otras personas, el espacio de vida temporal se extiende lejos en el futuro. Éstas se han puesto muchas más metas que sólo pueden ser realizadas en el futuro lejano. En comparación con las personas con una PTF corta, aquéllas con una PTF larga tienen relativamente muchas más metas de largo plazo que metas de corto plazo. En consecuencia, "la perspectiva de tiempo futuro evoluciona 
desde el establecimiento de la meta motivacional. Está formada por los relativamente distantes objetos-meta que son procesados por un individuo" (Nuttin \& Lens, 1985, p. 22).

La conceptualización de la PTF tiene una larga historia en la psicología, especialmente en Europa.

Para Lewin:

The setting of goals is closely related to time perspective. The goal of the individual includes his expectations for the future... $(1948, \text { p. } 113)^{2}$.

\section{Para Lersch:}

Wie die Vergangenheit, so ist andererseits auch die Zukunft in der Gegenwart des Erlebens enthalten. Jede erlebte Gegenwart ist Vorgriff in die Zukunft. Das gilt im dem Masse, als jeder Augenblick des seelischen Lebens durchwirkt ist von der Thematik und Dynamik seelischer Strebungen, die auf die Verwirklichung eines noch nicht bestehenden Zustandes gehen (1966, p. 47) ${ }^{3}$.

\section{Para Fraisse:}

The future only unfolds in so far as we imagine a future which seems to be realizable...There is no future without at the same time a desire for something else

2 "El establecimiento de metas está íntimamente relacionado con la perspectiva del tiempo. Las metas de un individuo incluyen sus expectativas para el futuro...”.

3 “Así como el pasado, también el futuro está contenido en el presente de la vivencia. Todo presente vivenciado es un adelanto del futuro. Esta apreciación tiene vigencia en la medida en la cual cada instante de la vida anímica está influido por la temática y la dinámica de las corrientes anímicas que se dirigen a la realización de un estado aún no existente". and awareness of the possibility of realizing it... (1963, pp. 172 y 174$)^{4}$.

\section{Para Nuttin:}

The psychological future is not just a learning effect of the past, it is essentially related to motivation... the future is the time quality of the goal object; the future is our primary "motivational space" $(1964, \text { p. } 63)^{5}$.

\section{Añade Nuttin:}

Le phénomène primaire de la direction vers le futur, tel qu'il se produit grâce au besoin, n'est qu'une orientation générale; elle se limite à un futur très immédiat, comme c'est le cas chez l'animal... C'est grâce au développement supérieur des fonctions cognitives et leur influence dans l'élaboration des besoins -notamment dans les processus de position de but... - que, chez l'homme, l'anticipation va se détacher graduellement de la situation actuelle et que des "perspectives" profondes vont s'ouvrir (1980, p. 13) $)^{6}$.

4 "El futuro sólo tiene lugar en la medida que imaginamos un futuro que parece ser realizable... No hay futuro $\sin$ que al mismo tiempo haya un deseo por algo y una conciencia de las posibilidades de realizarlo...".

5 "El futuro psicológico no es solamente un efecto de aprendizaje del pasado, se encuentra esencialmente relacionado con la motivación...el futuro es la cualidad de tiempo de la meta-objeto; el futuro es nuestro "espacio motivacional" primario".

6 "El fenómeno primario de la dirección hacia el futuro, tal como se produce por efecto de la necesidad, no es más que una orientación general; ella se limita a un futuro muy inmediato, como en el caso del animal... Es gracias al desarrollo superior de las funciones cognitivas y su influencia en la elaboración de las necesidades -especialmente en los procesos de establecimiento de metas... que, en el ser humano, la anticipación se va desligando gradualmente de la situación actual, y se van abriendo "perspectivas" profundas". 


\section{Para Gjesme:}

An individual's future time orientation (FTO) develops gradually to become a relatively stable personality characteristic in terms of a general capacity to anticipate and enlighten the future, including a cognitive elaboration of plans and projects and reflecting the degree of concern, involvement and engagement in the future $(1981, \text { p. } 125)^{7}$.

\section{Efectos motivacionales de la perspectiva de tiempo futuro}

Las diferencias individuales en la extensión (longitud y profundidad) de la PTF no resultan sólo del establecimiento de la meta motivacional; también tienen consecuencias motivacionales (De Volder y Lens, 1982; Gjesme, 1975; Lens, 1986; Raynor \& Entin, 1982). Ellas afectan la intensidad de la búsqueda de la meta presente, por ejemplo en la motivación estudiantil. Zaleski $(1987,1994)$ encontró que en comparación con individuos con una corta PTF, las personas con una larga PTF son más persistentes en el trabajo para una meta y obtienen más satisfacción de las acciones actuales orientadas hacia la meta. No sólo están motivadas

7 "La orientación del tiempo futuro (OTF) de un individuo se desarrolla gradualmente hasta convertirse en una característica relativamente estable de la personalidad en términos de la capacidad general para anticipar e iluminar el futuro, incluyendo la elaboración cognitiva de los planes y proyectos, y reflejando el grado de preocupación y compromiso en el futuro". hacia las metas distantes, sino también para las submetas próximas que conducen a una meta final (provisional) en el futuro distante.

Primero formularemos un modelo teórico (véase figura $\mathrm{N}^{\circ} 1$ ) que trata de explicar los efectos motivacionales de las diferencias individuales en la PTF y después lo aplicaremos a la motivación estudiantil. Los procesos subyacentes son conceptualizados en términos de la distancia psicológica hacia momentos futuros (Gjesme, 1982) y de los modelos de valor (modelos-VEI) expectativa $\mathrm{x}$ instrumentalidad $\mathrm{x}$ de la motivación humana (Feather, 1982).

\section{Perspectiva de tiempo futuro y distancia psicológica}

Se asume que los individuos con una PTF larga experimentan un intervalo dado de tiempo cronológico futuro (v.g., dos años), como más corto que las personas con una corta PTF. Esta relación entre la longitud de la PTF y la distancia psicológica hacia el futuro no será muy fuerte para intervalos de tiempo muy cortos (v.g., una hora, un día, una semana a partir de ahora), ni para intervalos muy largos hacia el futuro (v.g., 20 años a partir del presente) (véase figura $\mathrm{N}^{\circ} 2$ ). Aun para individuos con una PTF muy corta, una o dos semanas puede ser algo más bien cercano mientras que 20 o 30 años hacia el futuro puede ser algo muy lejano, incluso para personas con una PTF larga. Los intervalos de tiempo cronológico 
Figura № 1

Efecto motivacional de la perspectiva de tiempo futuro

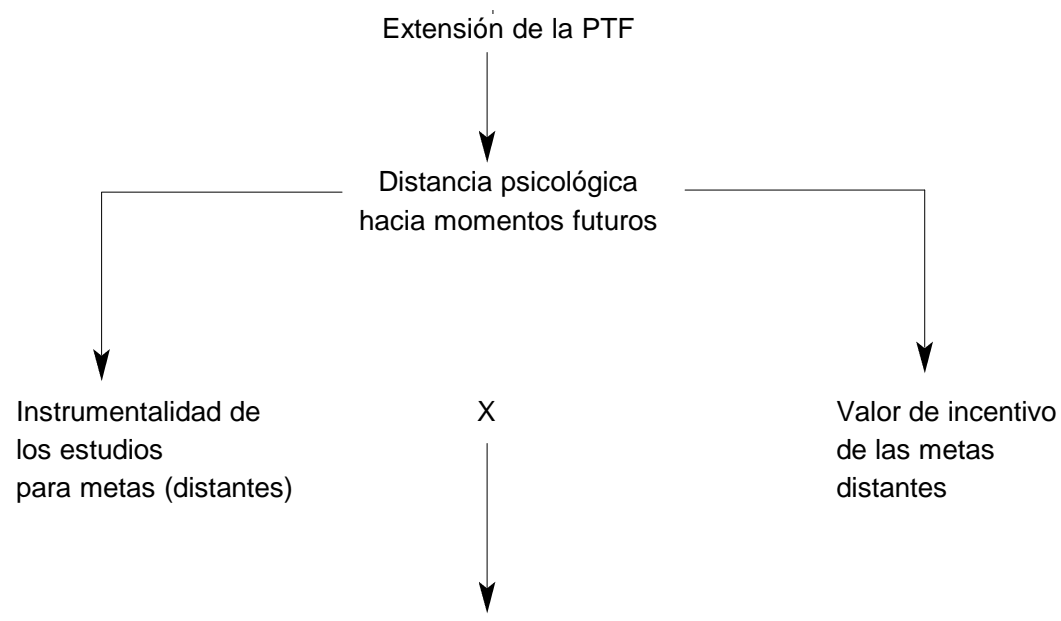

Motivación estudiantil

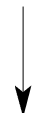

Consecuencias conductuales

en los que el efecto de las diferencias individuales en la PTF será de mayor intensidad, dependerá también de la edad.

Moreas y Lens (1991) pidieron a 245 estudiantes de noveno grado (14 a 15 años de edad) que dibujaran unas líneas que expresaran cuán larga era la experiencia del período de una semana, un mes, seis meses, un año y cinco años hacia el futuro. Este test de líneas validado (Cottle \& Pleck, 1969) asume que los individuos dibujarán líneas más largas cuando experimentan la distancia hacia un momento futuro (o la duración del intervalo de tiempo cronológico en- tre el momento presente y el futuro) como más largo. Cuanto más corta la línea, más corta la distancia psicológica. Como se esperaba, Moreas y Lens no encontraron correlaciones significativas entre la longitud de la PTF y la longitud de las líneas para intervalos cortos (una semana: .10; un mes .10) ni para un período largo de cinco años (.05). Para los intervalos de seis meses (.19) y un año (.15) las correlaciones son bajas pero estadísticamente significativas. El efecto de la PTF en la distancia psicológica es significativamente diferente para los intervalos de un mes y seis meses en comparación con los 
Figura № 2

Distancia psicológica hacia momentos en el futuro cercano, mediano y distante como una función de la longitud en la perspectiva del tiempo futuro individual.

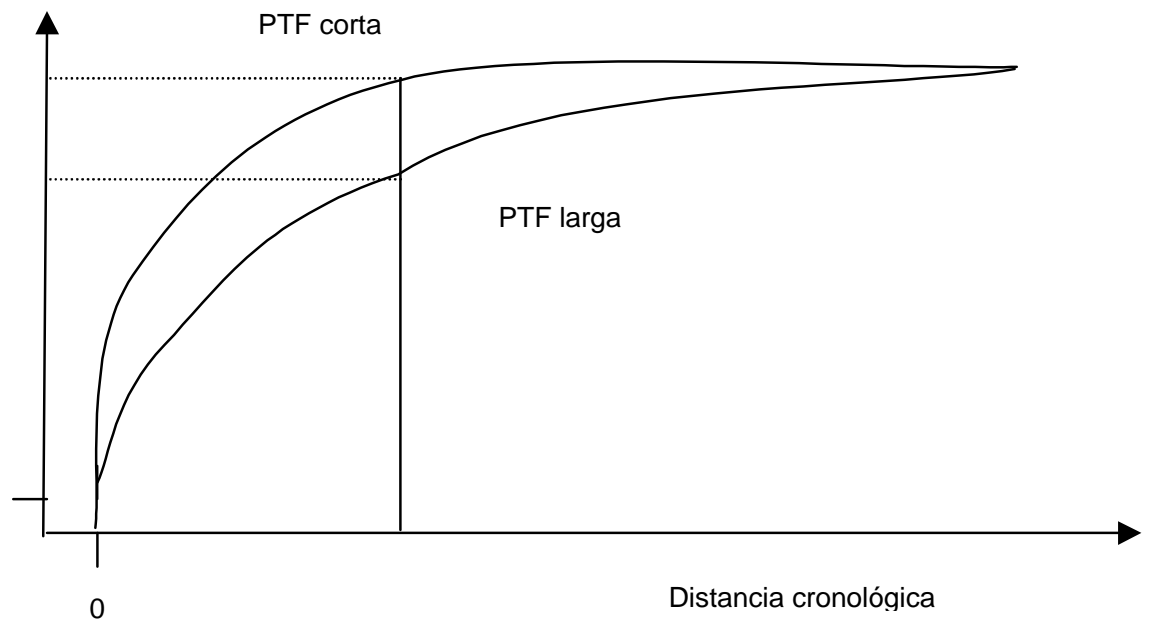

otros dos períodos (una semana y un año): $F(3,237)=3.65, p<.02$. Podemos concluir que los individuos con una PTF larga experimentan, en efecto, el futuro (intermedio) como más cercano que los individuos con una PTF más corta.

Perspectiva de tiempo futuro, instrumentalidad percibida y valor anticipado

De Volder y Lens (1982) distinguen entre el aspecto cognitivo y el aspecto dinámico de la PTF como una característica de la personalidad. El aspecto cognitivo puede ser entendido como una disposición para anticipar en el presente no sólo los efectos inmediatos de la acción potencial sino también sus consecuencias de largo plazo. Los estu-

diantes con una PTF larga pueden anticipar más fácilmente las implicancias de sus actividades de clase presentes para un futuro más distante (instrumentalidad) y así desarrollar estructuras conductuales medios-fin más duraderas. Esto incrementa la motivación instrumental del aprendizaje y los logros actuales en la escuela.

El aspecto dinámico de la PTF es concebido como una disposición de atribuir un alto valor a las metas, aun cuando ellas sólo puedan ser realizadas en un futuro más bien distante.

La investigación sobre la demora de la gratificación (Mischel, 1981) señala que el valor de incentivo de las recompensas (metas) decrece como una función de la longitud de su demora temporal. El valor de un objeto-meta dado 
disminuye con el incremento de la distancia temporal de tal objeto. Pero esta disminución es menos pronunciada para individuos con una PTF larga en comparación con individuos con una PTF corta. Puesto que la distancia psicológica hacia tales metas diferidas es menor para los individuos con una larga PTF, el valor de incentivo de las metas distantes será mayor cuanto más larga sea la PTF.

Como se muestra en la figura $\mathrm{N}^{\circ} 3$, el efecto de las diferencias individuales en la extensión de la PTF con respecto al valor del incentivo será menor para las metas cercanas o las metas muy distantes, que para las metas del futuro intermedio.

La investigación que valida "expectativa $\mathrm{x}$ instrumentalidad $\mathrm{x}$ modelos de valor" en la psicología motivacional
(Feather, 1982) ha mostrado que la intensidad de la motivación para una acción particular es una función multiplicativa del valor instrumental percibido que esa acción tiene para alcanzar resultados positivos, y el valor de incentivo de los resultados (metas).

De Volder y Lens (1982) encuentran que estudiantes de grado 11 (17-18 años de edad) altamente motivados atribuyen significativamente mayor valor a las metas situadas en el futuro relativamente distante que estudiantes menos motivados.

Estudiantes altamente motivados atribuyen también mayor valor a su trabajo escolar como medio para alcanzar metas en el futuro cercano y distante (véase tabla $\mathrm{N}^{\mathrm{o}} 1$ ).

Lens y Decruyenaere (1991) midieron el valor instrumental del estudio

Figura № 3

Valor de incentivo en diferentes distancias temporales como función de la extensión de la perspectiva de tiempo futuro.

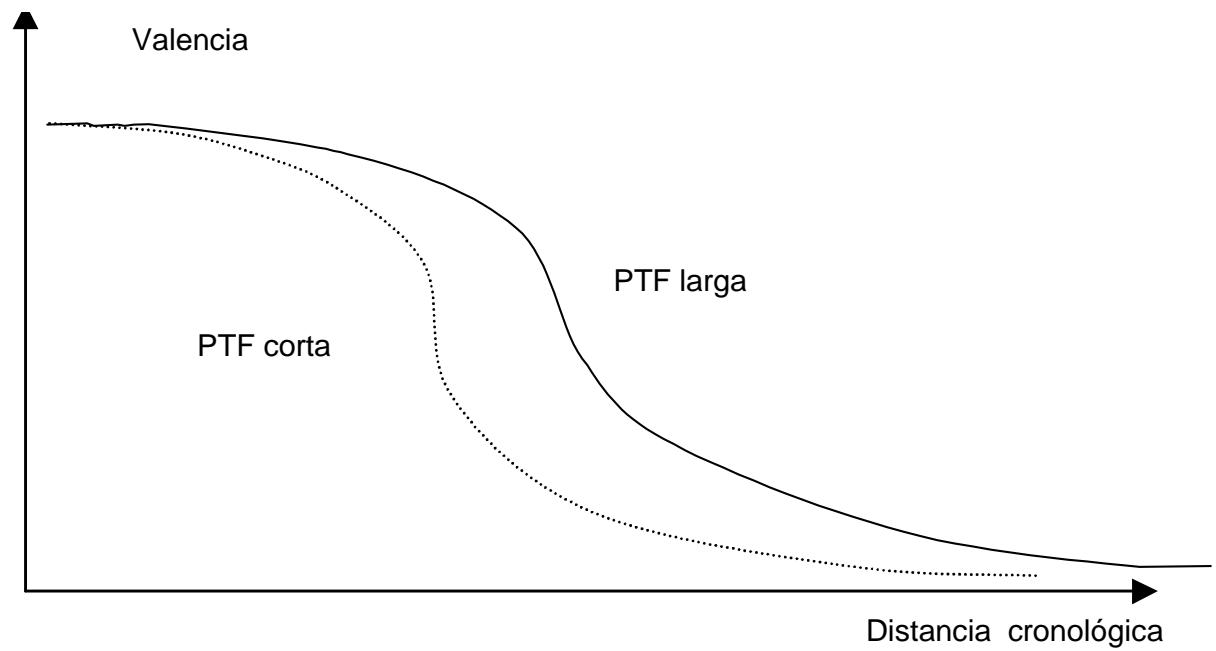


(en tres diferentes tipos de educación secundaria) en función al éxito posterior en la vida, como la diferencia entre la probabilidad de tal éxito como consecuencia de "esforzarse al máximo en la escuela" y la probabilidad de tener éxito sin esforzarse en la escuela. Los investigadores encontraron (véase tabla $\mathrm{N}^{\circ}$ 2) que en humanidades, en los niveles superior y básico de la educación técnica, los subgrupos de estudiantes con alta, mediana y baja motivación (basados en un cuestionario de medida) difieren significativamente (en la dirección predicha) en el valor instrumental o de utilidad de "esforzarse al máximo en la escuela" para lograr el éxito en la vida en general.

También Van Calster, Lens y Nuttin (1987) encontraron un efecto principal significativo de la instrumentalidad percibida sobre la motivación: Estudiantes en grados 11 y 12 (17-19 años de edad) que perciben su educación como importante para su futuro (alta instrumentalidad) están significativamente más motivados que sus pares que obtienen una puntuación baja para la instrumentalidad percibida (véase tabla $\mathrm{N}^{\mathrm{o}}$ 3). Van Calster et al. hallaron,

Tabla № 1

Valor promedio de las metas motivacionales en el futuro cercano y distante y el valor instrumental promedio de "esforzarse al máximo en la escuela" para alcanzar esas metas en el futuro cercano y distante para estudiantes con alta y baja motivación.

\begin{tabular}{l|cc|cc}
\hline $\begin{array}{l}\text { Motivación } \\
\text { estudiantil }\end{array}$ & \multicolumn{2}{|c|}{ Futuro cercano } & \multicolumn{2}{c}{ Futuro lejano } \\
Valor & Instrumentalidad & Valor & Instrumentalidad \\
\hline Alta & 7.24 & .31 & 6.74 & .61 \\
Baja & 7.22 & .11 & 6.54 & .29 \\
\hline
\end{tabular}

Tabla № 2

Puntuación promedio de la instrumentalidad para estudiantes con alta, mediana y baja motivación en humanidades y en los niveles básico y superior de la educación técnica.

\begin{tabular}{l|c|cc}
\hline $\begin{array}{l}\text { Motivación } \\
\text { estudiantil }\end{array}$ & Humanidades & \multicolumn{2}{|c}{$\begin{array}{c}\text { Educación técnica } \\
\text { Nivel básico }\end{array}$} \\
\hline & & & \\
Alta & 3.25 & 3.43 & 3.05 \\
Mediana & 2.99 & 3.18 & 2.92 \\
Baja & 2.41 & 2.37 & 2.26 \\
\hline
\end{tabular}


sin embargo, un significativo efecto interactivo de la instrumentalidad y la actitud afectiva hacia el futuro individual. La instrumentalidad percibida incrementa la motivación estudiantil pero sólo para aquellos estudiantes que tienen una actitud positiva hacia su futuro individual (véase los grupos de alta y mediana afectividad en la tabla $\mathrm{N}^{\circ} 3$ ). $\mathrm{El}$ atribuir una alta utilidad a los resultados escolares tiene el efecto opuesto en la motivación al estudio en los estudiantes con una visión negativa de su futuro. La combinación de una alta percepción de la instrumentalidad de "esforzarse al máximo en la escuela" para el futuro personal y una actitud afectiva altamente positiva hacia el mismo futuro, tiene un efecto positivo en la motivación y los resultados en la escuela. Una visión pesimista del futuro parece ser una variable desmotivante.

Moreas y Lens (1991) pidieron a estudiantes de noveno grado que calificaran la importancia (valor) de diez metas motivacionales que normalmente pueden ser alcanzadas dentro de un período de dos años (metas cercanas) y otras diez metas que sólo podían ser alcanzadas después de al menos dos años (metas distantes) en una escala de cuatro puntos, que va desde "sin ninguna importancia para mî” hasta "muy importante para mí". Las metas cercanas y lejanas cubren las mismas categorías de contenido motivacional. Para medir la instrumentalidad, se les preguntó a los sujetos: "Si me esfuerzo al máximo en mis estudios, entonces las posibilidades de que alcance... (la meta)... son mayores que si no hago el esfuerzo: ciertamente verdadero (cuatro puntos) - probablemente verdadero - algo verdadero - ciertamente no verdadero (1 punto)".

La motivación estudiantil se midió preguntando a los sujetos si estaban de acuerdo o no con el contenido de 19 ítems (v.g., creo que me esfuerzo más que la mayoría de mis compañeros de aula; frecuentemente me es difícil empezar a hacer mis tareas en casa). Doce

Tabla № 3

Puntajes promedio de la motivación para el estudio como

una función de la actitud afectiva hacia el futuro personal y la instrumentalidad percibida en las calificaciones escolares

\begin{tabular}{lcccc}
\hline Instrumentalidad & \multicolumn{3}{c}{ Actitud afectiva } & Total \\
\hline Percibida & Baja (-) & Mediana $(+)$ & Alta $(++)$ \\
Alta & & & & \\
Baja & 7.28 & 11.70 & 13.05 & 10.79 \\
\hline Total & 9.00 & 10.15 & 8.29 & 9.35 \\
\hline
\end{tabular}


ítems tuvieron un valor de más de .30 en el factor que razonablemente puede ser identificado con "persistencia de estudio". Los siete otros ítems tienen un alto valor en un factor que se denomina "eficiencia de estudio". Los dos factores tienen la correlación .14 $(\mathrm{N}=217, \mathrm{p}<.05)$. La persistencia de estudio correlaciona .84 y la eficiencia de estudio .63 con el puntaje total de la motivación estudiantil. Ésta es la suma total para los 19 ítems.

La perspectiva de tiempo futuro se midió calculando para cada individuo la proporción del número de metas ubicadas en el futuro cercano (dentro de los dos años) con respecto al número de metas situadas en el futuro distante (más allá del período de dos años). Tanto menor es aquella proporción, más larga es la PTF. Las metas se expresan espontáneamente en una serie de oraciones para completar en el Motivational Induction Method o M.I.M. (Nuttin, 1980; Nuttin \& Lens, 1985).

El análisis de la varianza reveló el efecto significativo esperado de la PTF en la motivación estudiantil $(\mathrm{F}(3,239)=4.07, \mathrm{p}<.01)$. Una comparación posterior (test de Duncan) muestra que los estudiantes con una larga PTF (cuartil más alto) están significativa-

Tabla № 4

Puntajes promedio para las diferentes medidas de criterio como una función de la longitud de PTF y los resultados de un análisis posterior de Duncan (promedios con una letra común no son significativamente diferentes).

\begin{tabular}{lllll}
\hline $\begin{array}{l}\text { Variable } \\
\text { dependiente }\end{array}$ & \multicolumn{4}{l}{ Longitud de la perspectiva de tiempo futuro } \\
Muy corta & Corta & Larga & Muy larga \\
\hline Motivación & $8.10 \mathrm{~A}$ & $8.32 \mathrm{~A}$ & $8.53 \mathrm{AB}$ & $9.96 \mathrm{~B}$ \\
& & & & \\
Valor (V) & & & & \\
-cercano & 2.39 & 2.37 & 2.41 & 2.40 \\
-distante & 2.40 & $2.46 \mathrm{AB}$ & $2.53 \mathrm{~B}$ & $2.54 \mathrm{~B}$ \\
& & & & \\
Instrumentalidad (I) & $1.73 \mathrm{~A}$ & $1.71 \mathrm{~A}$ & $1.89 \mathrm{~A}$ & $1.86 \mathrm{~A}$ \\
-cercana & $1.90 \mathrm{~A}$ & $1.93 \mathrm{~A}$ & $2.04 \mathrm{AB}$ & $2.17 \mathrm{~B}$ \\
-lejana & & & & \\
& & & & \\
Valor x instrumentalidad & 4.17 & 4.10 & 4.65 & 4.56 \\
-cercano & $4.66 \mathrm{~A}$ & $4.92 \mathrm{~A}$ & $5.27 \mathrm{AB}$ & $5.65 \mathrm{~B}$ \\
-distante &
\end{tabular}

Cercano $=1$ semana y 1 mes

Distante $=6$ meses y 1 año. 
mente $(\mathrm{p}<.05)$ más motivados que los estudiantes con una corta o muy corta PTF (véase tabla $\mathrm{N}^{\circ} 4$ ).

Se anticipaba que la longitud de la PTF tuviera un efecto positivo en el valor anticipado de las metas, en la instrumentalidad percibida del comportamiento de estudio para alcanzar metas futuras y, por lo tanto, en el producto del valor y la instrumentalidad. Se anticipaba también que estas relaciones fueran más intensas para las metas distantes en comparación con las más cercanas. El efecto-interacción anticipado de la PTF y el período de tiempo en los valores de las metas fue, en efecto, significativo: $F(3,244)=3.15, \mathrm{p}<.03$. Los dos análisis separados de varianza revelaron un efecto no significativo de la PTF en el valor de las metas cercanas $(F(3,244)<1.00)$ y un efecto significativo esperado de la PTF en el valor de las metas distantes $(\mathrm{F}(3,244)=3.97, \mathrm{p}<.01)$. El valor anticipado de las metas distantes fue significativamente mayor para los individuos con una larga o una muy larga PTF que para los individuos con una corta PTF.

En el caso de la instrumentalidad, el efecto-interacción de la PTF esperado y el período de tiempo es marginalmente significativo en la dirección esperada $(\mathrm{F}(3,244)=2.11, \mathrm{p}<.10)$ : la PTF tiene un efecto mayor en la instrumentalidad percibida para las metas distantes que para las metas cercanas. Los análisis de Duncan después de los
ANOVAs separados para cada período de tiempo no mostró diferencias significativas entre los cuatro promedios de la instrumentalidad percibida para las metas cercanas (véase tabla $\mathrm{N}^{\circ} 4$ ). Para las metas lejanas, sin embargo, la instrumentalidad percibida del comportamiento de estudio resultó ser significativamente mayor para los estudiantes con una muy larga PTF que para los individuos con una corta o muy corta PTF (véase tabla $\mathrm{N}^{\circ} 4$ ).

Para el producto "valor $\mathrm{x}$ instrumentalidad" el efecto-interacción de la PTF esperado y el período de tiempo es significativo: $\quad \mathrm{F}(3,252)=3.22, \quad \mathrm{p}<.03$. ANOVAs separados para los dos períodos de tiempo muestran un significativo efecto principal en la longitud de la PTF en el producto de la instrumentalidad y el valor para metas distantes $(\mathrm{F}(3,252)=4.76, \mathrm{p}<.003)$ y un efecto marginalmente significativo para las metas en el futuro cercano $(\mathrm{F}(3,252)=2.46, \mathrm{p}<.07)$. Para las metas distantes, este producto es significativamente mayor para las personas con una PTF muy larga que para las personas con una corta o muy corta PTF (véase tabla $\mathrm{N}^{\circ} 4$ ).

No se encontró la correlación esperada entre la distancia psicológica hacia el futuro y los puntajes del "valor $\mathrm{x}$ instrumentalidad" para metas de largo plazo $(\mathrm{N}=250, \mathrm{r}=-.023$,n.s.). Además, los análisis de Lisrel muestran que, al menos en estos datos, los efectos motivacionales de las diferencias individuales 
en la PTF sobre la valencia e instrumentalidad, no se encuentran influidos por la distancia psicológica percibida de las metas ubicadas en un futuro distante.

Lens y Creten (1998) estudiaron la función motivacional de la intrumentalidad/utilidad percibida en las escuelas vocacionales básicas. Muchos de sus estudiantes tienen considerables problemas motivacionales para el trabajo académico, especialmente en cursos teóricos como matemáticas, lengua e historia. Y muchos de ellos empezaron en un mayor nivel de educación secundaria (v.g. humanidades, matemáticas y ciencias) pero fracasaron. Se vieron obligados a reducir sus aspiraciones académicas, orientándose primero a una educación más técnica y finalmente al nivel más elemental del entrenamiento vocacional práctico. Asumiendo que es casi imposible crear nuevamente una motivación intrínseca para cursos teóricos, la única manera de motivarlos es a través de metas extrínsecas. Queríamos saber si tales estudiantes que sí perciben la relevancia futura de, por ejemplo, un segundo idioma, estaban más motivados para dicho curso. ¿Podrían estar motivados por las futuras contingencias del trabajo académico actual? La correlación entre la motivación y la utilidad percibida de los cursos para las metas de corto plazo es .14 ( $\mathrm{p}=.004 ; \mathrm{n}=733)$ y para metas de largo plazo es .19 $(\mathrm{p}=.0001 ; \mathrm{n}=733)$. Las correlaciones eran mayores para los hombres (.20) que para las mujeres (.12) y también mayores en $9^{\circ}$ grado (.19) y $10^{\circ}$ grado (.30) que para $11^{\circ}$ grado (.00) y $12^{\circ}$ grado (.04). La tabla $\mathrm{N}^{\circ} 5$ muestra los puntajes promedio para la motivación como función de la instrumentalidad percibida para las metas de corto y largo plazo. Los análisis de la varianza señalan el esperado efecto significativo de la instrumentalidad para metas cercanas y distantes.

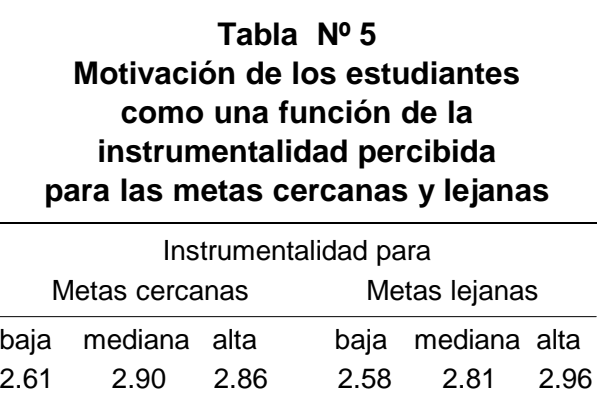

Los estudiantes para quienes el estudio tiene un valor instrumental bajo para las metas del futuro cercano y distante están menos motivados. Estos estudiantes atribuyen una mayor utilidad a sus cursos prácticos y están más motivados para ellos que para cursos teóricos o el curso de francés como segunda lengua (véase tabla $\mathrm{N}^{\circ}$ 6). Sin embargo, no están más motivados al francés que a otros cursos teóricos, aun cuando atribuyen una mayor importancia utilitaria al francés. A partir de otros datos de cuestionario hemos encontrado que en este mismo tipo de estudiantes la instrumentalidad o utilidad 
percibida es una condición importante pero no suficiente para estar motivado. Por ejemplo, los estudiantes saben que en Bélgica el francés es importante para su futura carrera profesional y para la vida en general, pero se quejan de que el contenido del curso y la forma en que se enseña no son muy motivadores.

\section{MOTIVACIÓN INTRÍNSECA Y ORIENTACIÓN FUTURA}

En la introducción sosteníamos que las metas de los estudiantes pueden estar situadas en las dimensiones "metas intrínsecas versus extrínsecas" y "metas inmediatas versus futuras". Un estudiante está intrínsecamente motivado si su aprendizaje y logros en la escuela constituyen una meta en sí misma. Las actividades que son extrínsecamente motivadas son aquellas que son instrumentales. Esto implica que las metas futuras o la perspectiva de tiempo futuro de los estudiantes sólo pueden crear motivación extrínseca. En la medida en que los estudiantes se encuentran motivados para el trabajo escolar por el valor de utilidad que éste posee para obtener otras metas importantes en el futuro cercano o lejano, ellos están -por definiciónextrínseca y no intrínsecamente motivados. Una importante pregunta teórica $\mathrm{y}$, ciertamente también práctica, es saber si tales metas futuras pueden afectar la motivación intrínseca de la misma manera negativa como lo hacen las recompensas extrínsecas y otras razones extrínsecas para estudiar. ¿Podría la perspectiva de tiempo futuro disminuir la motivación intrínseca para estudiar?

Primero resumiremos la investigación sobre la interacción entre las recompensas o metas extrínsecas y la motivación intrínseca. Luego discutiremos los efectos motivacionales de la utilidad o instrumentalidad de los diferentes tipos de metas futuras sobre la motivación intrínseca. Abordaremos primero el campo de la motivación de logro y luego las más recientes teorías de metas que, al menos parcialmente, han surgido de la teoría de la motivación de logro.

Tabla № 6

Instrumentalidad percibida promedio

(para el futuro cercano y lejano)

y motivación para tres diferentes tipos de cursos

\begin{tabular}{lccc}
\hline curso & \multicolumn{2}{c}{ instrumentalidad para } & motivación \\
& metas cercanas & metas lejanas & \\
\hline Teórico & 2.15 & 2.16 & 2.61 \\
Francés & 2.49 & 2.62 & 2.56 \\
Práctico & 2.53 & 2.89 & 3.21 \\
\hline
\end{tabular}




\section{Motivación intrínseca y recompensas extrínsecas}

Por muchos años se ha asumido que la motivación estudiantil -así como la motivación laboral- es la suma de los componentes intrínsecos y extrínsecos. Cada meta intrínseca y extrínseca puede ser concebida como una fuente adicional de motivación.

Sin embargo, la investigación básica en los últimos veinte años demuestra que las fuentes intrínsecas y extrínsecas de la motivación no son necesariamente aditivas. Las recompensas relevantes, prometidas, que son exógenas a la naturaleza del aprendizaje o de los logros, y que son dadas a unas actividades intrínsecamente motivadas, pueden disminuir la motivación intrínseca (Deci, 1975; Deci \& Ryan, 1985, 1992; Lepper \& Greene, 1978; Luyten \& Lens, 1981). Este fenómeno ocurre cuando se produce un efecto de sobrejustificación (demasiadas razones para realizar una tarea: el presente interés intrínseco y, por añadidura, la recompensa extrínseca prometida). Las recompensas y otras medidas de control causan un desvío en el locus de causalidad de lo interno a lo externo. Los individuos que se encuentran intrínsecamente motivados consideran su propio interés en la materia o en la tarea como la única razón para estudiarla o hacerla. Cuando reciben repetidamente recompensas extrínsecas, estas últimas son percibidas progresivamente como las razones para aprender y realizar correctamente. Ya no es el interés intrínseco sino las recompensas extrínsecas las que controlan las actividades. El interés intrínseco desaparece. Lepper y Greene (1978) se refieren a este fenómeno en el título de su libro como "Los costos escondidos de la recompensa".

No sólo las recompensas anunciadas pueden causar un cambio en el locus de la causalidad de lo interno a lo externo; también la evaluación, supervisión, coerción, presión de tiempo, etc. Todo ello reduce la motivación intrínseca y frecuentemente disminuye la calidad del desempeño.

Nuestra hipótesis es que el bajo nivel de motivación intrínseca en muchos estudiantes de secundaria se debe en parte a este efecto negativo de las diferentes recompensas extrínsecas que los estudiantes han recibido en la escuela primaria y secundaria por sus esfuerzos y altas calificaciones. Harter encontró “...un cambio sistemático y relacionado con las calificaciones de una orientación motivacional intrínseca en el tercer grado hacia una orientación más extrínseca en el noveno grado; el mayor cambio ocurre entre el sexto grado de educación primaria y el primero de la educación secundaria" (Harter \& Jackson, 1992, pp. 223-224).

La investigación meta-analítica más reciente demuestra sin embargo que "los efectos perjudiciales de la recompensa ocurren bajo condiciones altamente limitadas y fácilmente evita- 
bles..." (Eisenberg \& Cameron, 1996, p. 1153). Las recompensas extrínsecas no tienen este efecto negativo en la motivación intrínseca cuando son dadas de tal forma que su aspecto de control es menos prominente que su aspecto informativo. Las recompensas extrínsecas pueden ser dadas de tal manera que le pueden informar a los estudiantes cuán buenos son en las tareas por las que se les dan recompensas. En tal sentido, las recompensas refuerzan las percepciones y sentimientos de competencia, elevando la motivación intrínseca (Deci \& Ryan, 1985). Las recompensas extrínsecas “... pueden algunas veces complementar o incrementar la motivación intrínseca..." (Rigby, Deci, Patrick \& Ryan, 1992, p. 168). La motivación intrínseca, en consecuencia, no será disminuida si los individuos pueden mantenerse "auto-determinados aun si les ofrecen motivadores extrínsecos" (ídem).

Los estudiantes y trabajadores que no están intrínsecamente motivados en absoluto, pueden inclusive desarrollar un interés intrínseco en una actividad. En tanto estén sólo extrínsecamente motivados, la acción es el medio para obtener las recompensas extrínsecas. Actividades como el estudio, la carpintería, el navegar, correr, etc., pueden estar motivadas por metas extrínsecas tales como recompensas, ganarse la vida, mantenerse en buena salud. Después de un tiempo, estas mismas actividades pueden convertirse en algo in- trínsecamente interesante para el individuo. Una estudiante que resuelva problemas matemáticos porque su maestra o padres le han ofrecido una recompensa, puede descubrir que es buena en ello, que es divertido encontrar una solución a un problema que la desafía. Sus sentimientos de competencia serán reforzados y en consecuencia, se desarrollará un interés intrínseco por la matemática.

\section{El futuro en la motivación de logro}

La teoría original de Atkinson sobre motivación de logro (Atkinson, 1964; Atkinson \& Feather, 1966) no incluye el futuro como una variable motivacional. Su modelo episódico está referido a un solo episodio de logro. Está limitado (por principio) a la elección entre varias tareas de logro de diferentes niveles de dificultad. La intensidad de la motivación intrínseca de logro resultante para el éxito es una función de la necesidad individual de logro o del motivo para tener éxito y motivo para evitar el fracaso, o de la dificultad percibida de la tarea de logro o de la probabilidad del éxito y el valor incentivante de éste. Ninguno de estos aspectos se refiere al futuro.

Raynor (1969,1981; Raynor \& Entin, 1982) desarrolló la teoría de Atkinson para incorporar el concepto de instrumentalidad, utilidad o perspectiva de tiempo futuro (PTF) en la investigación de la motivación de logro. Su teoría es un modelo matemáticamente for- 
malizado que permite combinar la motivación intrínseca y la instrumental. Para Raynor, la instrumentalidad tiene un lugar central tanto para la orientación futura como para la motivación.

La orientación futura o la perspectiva del tiempo futuro es

... el impacto en la motivación para alguna actividad presente de la percepción de su relación instrumental, como un paso en una senda mayor hacia más distantes metas futuras y consecuencias arriesgadas" (Atkinson \& Raynor, 1974, p. 5).

Sin embargo, la teoría de Raynor sólo se aplica cuando la "actividad presente" es una tarea de logro y la "meta futura" es el éxito en otra tarea de logro, y las "consecuencias arriesgadas" son fracasos en aquellas tareas de logro futuras.

Raynor hace la distinción entre las tareas de logro aisladas sin referencia alguna al futuro, y dos tipos de tareas de logro: sendas contingentes y no-contingentes.

En una senda no-contingente de tareas de logro, el individuo prevé una larga o corta serie de tareas de logro consecutivas en las que él o ella puede tener éxito o fracasar. Pero el éxito en la primera tarea no es un requisito para luchar por el éxito en la siguiente tarea (v.g. una serie de exámenes finales). El éxito no tiene un valor instrumental o utilitario para el éxito futuro. La perspectiva del tiempo futuro en la lucha por el logro es operacionalizada por Raynor como el número de tareas de logro que son anticipadas por un individuo en un tiempo dado. Siguiendo la elaboración que hace Raynor de la teoría de Atkinson, el número de pasos o tareas en una senda no-contingente no tiene implicancias motivacionales. La intensidad de la motivación para luchar por el éxito en la primera tarea de una serie puede ser estimada de la misma forma que se hace para tareas de logro aisladas en la teoría original de la motivación de logro.

En una senda contingente de tareas de logro, el éxito en la primera tarea es una condición necesaria para intentar el éxito en la siguiente tarea, y así sucesivamente. El fracaso significa que uno no puede continuar la serie, que uno no puede luchar por el éxito en las siguientes tareas. El primer paso (tarea) debe ser dominado antes de poder continuar al segundo, que, a su vez, debe ser dominado para continuar al tercero, y así sucesivamente. Este tipo de perspectiva del tiempo futuro intensifica la motivación intrínseca de logro. Tanto más larga sea la senda contingente (tanto más tareas de logro incluya), tanto más intensa será la motivación intrínseca de logro resultante de la primera tarea de esa senda contingente (positiva para la orientación al éxito y negativa para sujetos con ansiedad de examen). La motivación para luchar por el éxito y evitar el fracaso en una tarea que es parte de una senda contingente de tareas de logro, es no sólo una función del motivo del individuo para 
tener éxito y motivo para evitar el fracaso y de la dificultad de tal tarea, sino también una función de su utilidad o instrumentalidad para obtener oportunidades para luchar por el éxito en tareas de logro futuras.

Es importante tener en cuenta, sin embargo, que en la teoría de Raynor las metas inmediatas y las metas futuras, que afectan la motivación actual, pertenecen a la misma categoría motivacional, la motivación intrínseca de logro. En una senda contingente, el individuo está motivado a tener éxito en la tarea, no sólo porque ello le da una satisfacción intrínseca (el orgullo del logro), sino también porque crea la oportunidad de luchar por éxitos siguientes y enorgullecerse de los logros. Para Raynor, en contraste con ciertos "teóricos intrínsecos", la motivación para la tarea actual no disminuye si la persona percibe la tarea como instrumental para metas de logro futuras. Todo lo contrario, percibir las tareas como instrumentales, incrementa la motivación.

Para Gjesme (1981), la teoría original de Raynor acerca de la longitud de la senda contingente y la obtención de una meta futura (probabilidad de alcanzarla) sólo contempla uno de los diversos factores que influyen en la distancia a la meta percibida en el tiempo. La distancia psicológica hacia la meta futura es, en efecto, “... una función negativa de la probabilidad (expectativa) de alcanzar la meta futura" (Gjes- me, 1981, p. 120). Pero es, a su vez, una función lineal positiva de la distancia objetiva de la meta en el tiempo" (Gjseme, 1981, p. 124).

Más adelante, Raynor (1981) hace una distinción entre la "jerarquía de tareas" y la "jerarquía de tiempo". La jerarquía de tareas se refiere al número de tareas de logro en una senda contingente. La teoría original de Raynor analiza cómo esta jerarquía afecta la motivación para la primera tarea de logro en una senda contingente. La jerarquía de tiempo se refiere a la duración temporal anticipada de una senda contingente o de la distancia cronológica hacia las metas de logro. Tanto mayor sea la distancia cronológica, mayor será la distancia psicológica. Los efectos motivacionales de las jerarquías de tarea y de tiempo son opuestos. Basándose en las hipótesis sobre gradiente de metas de Miller, Raynor asumió y encontró (Raynor \& Entin, 1982), como Gjesme (1975), que la motivación de logro resultante típica será más intensa cuando la longitud cronológica de la senda es más corta.

La idea básica de Raynor es que cada uno de los pasos futuros proporciona una contribución motivacional a la motivación en la tarea actual. Tanto más larga la senda, mayor la contribución a la motivación; pero al mismo tiempo, tanto más distante es el paso de la tarea actual, menor será la contribución de ese paso individual. Esto significa que los individuos orientados al 
éxito están más intensamente motivados a luchar por el éxito en el primer paso de una senda contingente cuando hay más tareas de logro en un menor intervalo de tiempo cronológico. En el caso de individuos con el temor al fracaso, se aplica lo contrario. Estos últimos serán más inhibidos. Aún más, los datos empíricos de Gjesme (1974, 1976) muestran que “... la aparición de motivos se incrementa conforme la distancia a la meta en el tiempo decrece" (Gjesme, 1981, p. 124). Pero más importante que la distancia cronológica hacia la meta de logro futura, es la duración subjetivamente anticipada de ese intervalo de tiempo (Gjesme, 1975). Y esto depende de la perspectiva del tiempo futuro individual o de la orientación del tiempo futuro.

\section{EL FUTURO Y LA ORIENTACIÓN DE LA} META INTRÍNSECA ${ }^{8}$

\section{De la teoría de la motivación de logro a la teoría de meta}

La meta del motivo de logro es ser exitoso en la competencia con algún estándar de excelencia. La competencia es para McClelland, Atkinson, Clark y Lowell (1953): “... quizás más clara cuando uno de los individuos se encuentra en una actividad competitiva (otra diferente a la pura agresión) en donde ganar o hacer algo tan bien o

8 Véase también Lens \& Rand (1997). mejor que otro individuo es el fin esencial" (ibíd., p. 111). Añaden, sin embargo, que tal competencia es también evidente “... en la preocupación... de cuán bien la tarea específica está siendo hecha, independientemente de lo bien que lo esté haciendo otra persona" (ídem). El estándar de excelencia no implica una competencia con otras personas, sino el satisfacer requerimientos autoimpuestos para un buen desempeño. También se considera que el que uno se encuentre involucrado en logros únicos o extraordinarios tales como inventos, creaciones artísticas y descubrimientos científicos, expresa la necesidad de logro, aun cuando no se mencione un estándar de excelencia.

La definición operacional tradicional de la necesidad de logro o del motivo para tener éxito hace una distinción entre dos tipos de metas de logro, como una función de los criterios o estándares de excelencia que son usados para evaluar el resultado del desempeño como éxito o fracaso: las normas individuales versus las sociales. Pero estos diferentes tipos de metas son percibidos como expresiones de la misma necesidad de logro.

Sin embargo, en una teoría de meta más reciente (Ames, 1992; Dweck, 1986, 1991; Maehr, 1976; Maehr \& Midgley, 1996; Maehr \& Pintrich, 1991; Nicholls, 1984; Pintrich \& Schunk, 1996) estos dos diferentes tipos de "metas de logro" se comprenden como pertenecientes a dos tipos muy 
diferentes de motivación. Se usan varias etiquetas para referirse a estas orientaciones de metas. Preferimos llamarlas "metas de aprendizaje o metas de tareas" versus "metas de desempeño o metas de logro". Estas metas corresponden a las dos actividades principales que se esperan de los estudiantes en la escuela: aprendizaje versus desempeño/logro.

Cuando los estudiantes están involucrados en actividades de aprendizaje, su meta es incrementar su conocimiento y competencias, entender y dominar asuntos cada vez más complejos. En tales tareas no pueden fracasar sino sólo mejorar. En estas actividades no aparecen ni la ansiedad debilitante de los exámenes ni el temor al fracaso. En las tareas de aprendizaje, los estudiantes están orientados hacia las tareas. Su meta principal es entender un problema y encontrar la forma de resolverlo. Para evaluar los resultados del aprendizaje se utilizan las características objetivas de la tarea y las normas individuales. Este tipo de orientación-hacia-la-tarea corresponde a la motivación intrínseca que resulta de la curiosidad o la necesidad de conocimiento e información, y la necesidad de dominio, competencia y eficiencia en la resolución de tareas desafiantes.

Cuando un estudiante rinde un examen tiene como meta obtener el éxito académico y evitar el fracaso. Este tipo de tareas de logro o desempeño suscita una egoorientación. En este caso, el in- dividuo se preocupa más en lo buena o mala que será su actuación frente a otros individuos y se ocupa menos de la tarea en sí. Prevalecen las normas o criterios sociales. El objetivo de los estudiantes es mostrarse a sí mismos y a otros (maestros, padres y pares) cuán buenos son. Si no se encuentran entre los mejores de su clase, su principal preocupación será evitar la percepción de incompetencia (tanto en sus propios ojos como en los de otras personas importantes). De una manera auto-defensiva, tales estudiantes evitan tareas demasiado desafiantes o no le ponen suficiente esfuerzo, puesto que la combinación de un gran esfuerzo y bajos resultados (anticipados) podría llevarlos a la inferencia de poseer una habilidad inferior.

\section{Orientación futura en la teoría de metas}

La teoría de metas no toma en cuenta los efectos motivacionales de la instrumentalidad o de la orientación futura. Implícitamente asume que los individuos egoorientados $\mathrm{u}$ orientados hacia el desempeño, y ciertamente individuos orientados hacia la tarea, están concentrados en el presente. El orientarse hacia la tarea o el estar intrínsecamente motivado para una tarea parece implicar que uno tiene que estar totalmente absorbido por la tarea en cuestión. Esto se explica muy bien con la descripción que hace Csikszentmihalyi (1990) de la "experiencia de flujo". 
En la vida diaria, sin embargo, muchos estudiantes y trabajadores intrínsecamente motivados se encuentran al mismo tiempo profundamente involucrados en sus carreras profesionales. No sólo están absorbidos con sus tareas presentes sino que además muchos de ellos están orientados hacia el futuro. Combinan las metas intrínsecas con la instrumentalidad. Como afirmamos en la introducción, la escuela no es sólo un fin en sí mismo, ella también se orienta hacia el futuro. Aprender y obtener buenas calificaciones puede ser divertido en sí mismo, pero también es importante para alcanzar otras metas académicas y profesionales en el futuro que puedan ser más desafiantes. Los trabajadores intrínsecamente motivados pueden estar también fuertemente motivados para hacer una carrera en su vida profesional. La combinación de la dimensión "orientación-hacia-la-tarea versus orientación-hacia-el-logro" y la dimensión temporal "orientación-presente versus orientación-futura" resulta en cuatro tipos generales de impulso motivacional. La teoría de Atkinson de la motivación intrínseca de logro se aplica para las tareas de logro sin instrumentalidad ni orientación-futura (tipo 1). Raynor y Gjesme trabajaron esta teoría para integrar la orientaciónfutura en la motivación de logro. Pero en sus teorías, las metas presentes y futuras se limitan a éxitos intrínsecamente satisfactorios en las tareas de logro (tipo 2). Debemos enfatizar que la "meta de logro" tiene un significado más amplio en la teoría de Atkinson que en las más recientes teorías de metas. Cuando las metas de tareas se discuten en estas teorías de metas, se hace sin tomar en cuenta (al menos explícitamente) las consecuencias futuras de la tarea (tipo 3). La actividad es un fin en sí mismo, es "juego", no "trabajo” (Lepper \& Cordova, 1992).

Pero de la misma forma que en la tarea de logro, la tarea de aprendizaje puede ser un medio para un fin, ciertamente en el entorno de la clase real. ¿Qué significa, motivacionalmente hablando, estar orientado-hacia-la-tarea y ver, al mismo tiempo, la utilidad o instrumentalidad de la tarea para otras metas (tipo 4)? Eso es lo que veremos a continuación.

Primero queremos hacer una distinción entre dos tipos diferentes de intrumentalidad de las metas de tarea, una distinción que no es nueva en psicología motivacional. Las metas de tarea pueden ser combinadas con a) metas extrínsecas inmediatas o diferidas (v.g., recompensas) u otras variables de control (tipo 4a), y b) otras metas de tarea en el futuro cercano o distante (tipo 4b).

La combinación de la orientación a la tarea o motivación intrínseca (en general) y las metas extrínsecas inmediatas o recompensas (u otras variables de control) ha recibido mucha atención en la investigación teórica y empírica. Hemos discutido arriba esta línea de in- 
vestigación. Desde un punto de vista conceptual, no hay mucha diferencia si las recompensas extrínsecas son inmediatas o diferidas. En ambos casos hay instrumentalidad, motivación extrínseca y regulación externa. Para Deci y Ryan (1985) una actividad es externamente regulada si está controlada por las condiciones que son claramente externas al individuo. La meta se ubica fuera de la actividad misma. El acto es meramente instrumental y no está controlado por una meta autónoma sino por la condición externa (v.g., incentivos, supervisión, evitar castigo, plazos, presiones).

¿Qué clase de instrumentalidad está presente en el tipo $4 \mathrm{~b}$ ? Una estudiante de secundaria puede estar altamente motivada para sus estudios en general, y para algunos cursos en particular (v.g., matemáticas, física, geometría), no sólo porque ella está intrínsecamente motivada y orientada-hacia-la-tarea, sino porque tiene planes de ir a la universidad y convertirse en una ingeniera civil. Esto es lo que ella decidió para sí misma. ¿En qué forma esas metas académicas y profesionales afectan su motivación intrínseca actual?

Queremos hacer una distinción entre tres diferentes subcategorías de instrumentalidad, como una función de la(s) meta(s) percibida(s) de la estudiante en cada momento en el tiempo.

En un primer escenario, si la joven estudia mucho sólo porque quiere llegar a ser una ingeniera para ganar mu- cho dinero y tener un posición acomodada, entonces ella sólo está extrínsecamente motivada. Éste es un ejemplo del tipo 4a. La tarea de aprendizaje y las metas futuras no pertenecen a la misma categoría motivacional. En tales casos, el comportamiento de estudio es regulado externamente y la instrumentalidad puede recortar la curiosidad intrínseca y la necesidad para la competencia y eficacia.

En un segundo caso, las metas actuales en la secundaria y las metas de tareas futuras como estudiante universitaria y después como ingeniera, pueden pertenecer a la misma categoría de contenido: adquirir, desarrollar y aplicar tipos particulares de conocimiento y tecnología. Esta situación es bastante análoga a la senda contingente de Raynor de las tareas de logro intrínsecamente motivadas. Las mismas tareas, las mismas competencias o sentimientos de competencia y eficacia pueden estar en juego en las diferentes fases de la carrera. Sus metas están al mismo tiempo dentro y fuera de las tareas inmediatas de aprendizaje, pero todas ellas están dentro de la tarea más amplia de tener muy buena base en matemática, física, construcción, etc., o, en otras palabras, tener los fundamentos de una buena ingeniera civil. Sostenemos que este tipo de instrumentalidad $u$ orientación futura no disminuirá sino, al contrario, aumentará la fuerza de la motivación intrínseca para las matemáticas, física, etc. 
Tercero, nuestra estudiante tiene cursos tales como historia, biología, etc., que no están directamente relacionados con sus posteriores metas educativas y profesionales, pero son cursos obligatorios. Ella se encuentra positivamente motivada también para esos cursos, pero ha decidido que quiere convertirse en una ingeniera egresada de una universidad prestigiosa. Ésta es una meta muy importante en su vida. En el presente se ve a sí misma como una "futura ingeniera". Por lo tanto necesita altas calificaciones. ¿Significa que este tipo de instrumentalidad hace extrínseca su motivación para estos cursos? Sí. ¿Se consideraría que está externamente regulada? No.

Heyman y Dweck (1992) probablemente llamarían a esto una motivación adaptativa y óptima. La estudiante combina la motivación intrínseca con metas futuras importantes. Si sólo estudia cursos en los que está intrínsecamente interesada, probablemente ella nunca ingresaría a una universidad prestigiosa para convertirse en ingeniera.

Deci y Ryan originalmente crearon un dilema para ellos mismos caracterizando todo comportamiento instrumental como motivado extrínsecamente y regulado externamente, identificando la mayoría de las planificadas secuencias de comportamiento de largo plazo reguladas volitivamente y que conducen a metas futuras, incluyendo las metas autoimpuestas, como activi- dades inferiormente motivadas. Más tarde resolvieron el problema distinguiendo cuatro tipos de regulación externa o "grados de autodeterminación" (Deci \& Ryan, 1985, 1992; Rigby, Deci, Patrick \& Ryan, 1992).

La regulación externa se refiere a las actividades que están reguladas por las condiciones que son claramente externas al individuo (v.g., la promesa de una recompensa, el temor al castigo, una orden). El primer paso hacia la internalización e integración se denomina regulación introyectiva. El comportamiento es motivado por las presiones internas que no son consonantes con el propio individuo. La persona misma siente que debe hacerlo, si no, sentirá vergüenza, remordimiento, mala conciencia, etc. La presión es interna pero extraña al yo, y por lo tanto, representa una regulación controlada externamente (locus de control externo, no autodeterminación). Regulación identificada es el nombre que se usa para el nivel en donde la actividad tiene lugar, no porque uno sienta que debiera hacerlo, sino porque es aceptada por el individuo como un paso hacia una meta personalmente importante. Esto es posible cuando una persona se identifica con un valor subyacente a dicha meta, y consiguientemente a la actividad, y ha aceptado la regulación como propia. El comportamiento es instrumental, y por lo tanto, es motivado extrínsecamente. Pero es instrumental para obtener la meta autodetermina- 
da, y, por lo tanto, más bien autónoma. Este tipo de regulación se aplica a la estudiante arriba mencionada. La regulación integrada es para Rigby, Deci, Patrick y Ryan, "la forma más autónoma y autodeterminada de la motivación extrínseca y resulta de la integración... de identificaciones separadas dentro del sentido coherente del propio yo" (1992, p. 70). Del mismo modo que la motivación intrínseca, ella representa una base para el funcionamiento autodeterminado. Mientras que los comportamientos intrínsecamente motivados son autotélicos o una meta como tal, los comportamientos integrados son instrumentales. Pero ambos tipos de comportamientos son regulados internamente y controlados por el individuo. Rigby et al. (1992, p. 171) suponen que la regulación integrada conduzca a una más alta calidad de aprendizaje y experiencia que aquellas que son resultado de los procesos regulatorios externos e introyectivos. Nuestra hipótesis es que también con la motivación extrínseca pero combinada con la regulación integrada, la calidad del aprendizaje no dista mucho del aprendizaje motivado intrínsecamente. Esto implica que, referidos a sus efectos cualitativos en el proceso de aprendizaje, los cuatro grados de regulación externa pueden ser agrupados en dos categorías cualitativas: regulación externa e introyectiva versus regulación identificada e integrada.

\section{Conclusión}

Se ha propuesto la hipótesis de que no hay nada incorrecto, ni desde un punto de vista motivacional, ni por la calidad de las experiencias de aprendizaje, respecto de dos importantes y muy comunes tipos de motivación instrumental que deriva de las metas futuras educacionales y profesionales de los estudiantes. Algunas metas futuras educacionales y profesionales pueden ser identificadas como metas de tareas que pertenecen a la misma categoría de contenido que las metas de tarea actuales en la escuela. La motivación instrumental que ellas crean no es en realidad una motivación extrínseca, como es usual en la motivación instrumental. Es más bien una motivación intrínseca de tarea, análoga a la noción de instrumentalidad en una senda contingente de logros de tarea de Raynor.

La escolaridad es muy importante para alcanzar las metas profesionales autodeterminadas. El segundo tipo de instrumentalidad en la educación, en efecto, crea motivación extrínseca. Sin embargo, puesto que tales metas personales importantes no se experimentan como reguladores externos sino como fuentes de "regulación identificada", este tipo de instrumentalidad no disminuirá la ya presente orientación intrínseca de tarea en la escuela, ni debilitará el aprendizaje de alta calidad. Pero, se necesita en esto también más investigación. 


\section{REFERENCIAS}

Ames, C. (1992). Classrooms: goals, structures, and student motivation. Journal of Educational Psychology, 84, 261271.

Ames R.E., \& Ames , C. (Eds.) (1984). Research on motivation in education. Student motivation (Vol.1). Orlando: Academic Press.

Atkinson, J.W. (1964). An introduction to motivation. Princeton: Van Nostrand.

Atkinson, J.W., \& Feather, N.T. (Eds.) (1966). A theory of achievement motivation. New York: Wiley.

Atkinson, J.W., \& Raynor, J.O. (1974). Introduction and overview. En J.W. Atkinson \& J.O. Raynor (Eds.), Motivation and achievement (pp. 3-11). Washington, DC: Winston \& sons.

Cottle, J.T., \& Pleck, J.H. (1969). Linear estimations of temporal extensions: The effect of age, sex, and social class. Journal of Projective and Personality Assessment, 33, 81-93.

Csikszentmihalyi, I. (1990). Flow. The psychology of optimal experience. New York: Harper \& Row.

Deci, E.L. (1975). Intrinsic motivation. New York: Plenum.

Deci, E.L. \& Ryan, R.M. (1985). Intrinsic motivation and self determination in human behavior. New York: Plenum Press.

Deci, E.L., \& Ryan, R.M. (1992). The initiation and regulation of intrinsically motivated learning and achievement. En A.K. Boggiano \& T.S. Pittman, (Eds.), Achievement and motivation: A social-developmental perspective (pp.
9-36). Cambridge: Cambridge University Press.

De Volder, M., \& Lens, W. (1982). Academic achievement and future time perspective as a cognitive-motivational concept. Journal of Personality and Social Psychology, 42, 566-571.

Dweck, C.S. (1986). Motivational processes affecting learning. American Psychologist, 41, 1040-1048.

Dweck, C.S. (1991). Self-theories and goals: Their role in motivation, personality and development. Nebraska Symposium on Motivation, 38, 199-235.

Eisenberger, R., \& Cameron, J. (1996). Detrimental effects of reward: Reality or myth? American Psychologist, 51, 1153-1166.

Feather, N.T. (1982). Expectations and actions: expectancy-value models in psychology. Hillsdale, NJ: Erlbaum.

Fraisse, P. (1963). The psychology of time. Westport: Greenwood.

Gjesme, T. (1974). Goal distance in time and its effects on the relation between achievement motives and perfomance. Journal of Research in Personality, 8, 161-171.

Gjesme, T. (1975). Slope of gradients for performance as a function of achievement motive, goal distance in time, and future time orientation. Journal of Psychology, 91, 143-160.

Gjesme, T. (1976). Future-time gradients for perfomance in test anxious individuals. Perceptual and Motor Skills, 42, 235-242.

Gjesme, T. (1981). Is there any future in achievement motivation? Motivation and Emotion, 2, 115-138. 
Gjesme, T. (1982). Psychological goal distance: The lost dimension in achievement motivation research. En W. Hacker, W. Volpert, \& M. von Cranach (Eds.), Cognitive and motivational aspects of action (pp. 86-98). Amsterdam: North-Holland.

Harter, S. (1981). A new self-report scale of intrinsic versus extrinsic orientation in the classroom: Motivational and informational components. Developmental Psychology, 17, 300-312.

Harter, S., \& Jackson, B.K. (1992). Trait vs. nontrait conceptualizations of intrinsic/extrinsic motivational orientations. Motivation and Emotion, 16, 209-230.

Heyman, G.D. \& Dweck, C.S. (1992). Achievement goals and intrinsic motivation: Their relation and their role in adaptive motivation. Motivation and Emotion, 16, 231-247.

Lens, W. (1986). Future time perspective: A cognitive-motivational concept. En D.R. Brown \& J. Veroff (Eds.), Frontiers of motivational psychology (pp. 173-190). New York: Springer-Verlag.

Lens, W., \& Creten, H. (1997). Manuscript in preparation.

Lens, W., \& Decruyenaere, M. (1991). Motivation and demotivation in secondary education: Student characteristics. Learning and Instruction, 1, 145-159.

Lens, W., \& Moreas, M.-A. (1994). Future time perspective: An individual and a societal approach . En Z. Zaleski (Ed.), Psychology of future orientation (pp. 23-38). Lublin: Towarzystwo Naukowe KUL.

Lens, W., \& Rand, P. (1997). Combining intrinsic goal orientations with profes- sional instrumentality/utility in student motivation. Polish psychological Bulletin, 28, 103-123.o

Lepper, M.R., \& Cordova, D.I. (1992). A desire to be taught: Instructional consequences of intrinsic motivation. Motivation and Emotion, 16, 187-208.

Lepper, M.R., \& Greene, D. (Eds.) (1978). The hidden costs of reward. Hillsdale, NJ: Erlbaum.

Lersch, Ph. [1966]. Aufbau der Person. München: Johann Ambrosius Barth.

Lewin, K. (1942). Time perspective and morale. En G. Watson (Ed.), Civilian morale. Boston: Houghton Mifflin.

Lewin, K. (1948). En G.W. Lewin (Eds.), Resolving social conflicts. Selected papers on group dynamics. New York: Harper \& Brothers.

Luyten, H., \& Lens, W. (1981). The effect of earlier experience and reward contingencies on intrinsic motivation. $\mathrm{Mo}$ tivation and Emotion, 5, 25-36.

Maehr, M.L. (1976). Continuing motivation: An analysis of a seldom considered educational outcome. Review of Educational Research, 46, 443-462.

Maehr, M.L., \& Midgley, C. (1996). Transforming school cultures. Boulder: Westview Press.

Maehr, M.L., \& Pintrich, P.R. (1991). Advances in motivation and achievement: Goals and self-regulation (Vol. 7). Greenwich, CT: JAI Press.

Malone, Th. W., \& Lepper M.R. (1987). Making learning fun: a taxonomy of intrinsic motivations for learning. En R.E. Snow \& M.J. Farr (Eds.), Aptitude, learning, and instruction. Conative and 
affective process analyses (Vol.3 pp. 223-253). Hillsdale, Nj: Erlbaum.

McClelland, D. C., Atkinson, J.W., Clark, R.A., \& Lowell, E.L. (1953). The achievement motive. New York: Appleton-Century-Crofts

Mischel, W. (1981). Objective and subjective rules for delay of gratification. En G.d'Ydewalle \& W. Lens (Eds.), Cognition in human motivation and learning (pp. 33-58). Leuven \& Hillsdale, NJ: Leuven University Press \& Erlbaum.

Moreas, M.-A. \& Lens W. (1991). De motivationele betekenis van het individueel Toekomstperspectief - project OT/88/6 (3 vols.) [The motivational significance of the individual future time perspective: an unpublished research report]. K.U. Leuven: Departamento de Psicología, sin publicar.

Nicholls, J.G. (1984). Achievement motivation: Conceptions of ability, subjective experience, task choice, and performance. Psychological Review, 91, 328-346.

Nuttin, J. (1964). The future time perspective in human motivation and learning. In Proceedings of the 17 th International Congress of Psychology (pp. 6082).Amsterdam: North-Holland

Nuttin, J. (1980). Motivation et perspectives d'avenir. Leuven: Presses Universitaires de Louvain.

Nuttin, J. (1984). Motivation, planning, and action. A relational theory of behavior dynamics. Leuven \& Hillsdale, NJ: Leuven University Press \& Erlbaum.

Nuttin, J., \& Lens, W. (1985). Future time perspective and motivation: Theory and research method. Leuven \& Hillsdale, NJ: Leuven University Press \& Erlbaum.

Pintrich, P.R., \& Schunk, D. (1996). Motivation in education: Theory, research, and applications. Columbus, $\mathrm{OH}$ : Prentice-Hall/Merrill College.

Raynor, J.O. (1969). Future orientation and motivation of immediate activity: An elaboration of the theory of achievement motivation. Psychological Review, 76, 606-610.

Raynor, J .O. (1981). Future orientation and achievement motivation: Toward a theory of personality functioning and change. En G. d'Ydewalle \& W. Lens (Eds.), Cognition in human motivation and learning (pp. 199-231). Leuven \& Hillsdale, NJ: Leuven University Press \& Erlbaum.

Raynor, J.O., \& Entin, E.E. (1982). Motivation, career striving, and aging. Washington, DC: Hemisphere.

Rigby, C. S., Deci, E.L., Patrick, B.C. \& Ryan, R.M. (1992). Beyond the intrinsic-extrinsic dichotomy: Self-determination in motivation and learning. Motivation and Emotion, 16, 165-185.

Van Calster, K., Lens, W., \& Nuttin, J. (1987). Affective attitude toward the personal future: Impact on motivation in high school boys. American Journal of Psychology, 100, 1-13.

Zaleski, Z. (1987). Behavioral effects of self-set goals for different time ranges. International Journal of Psychology, 22, 17-38.

Zaleski, Z. (Ed.) (1994). Psychology of future orientation. Lublin Towarzystwo Naukowe KUL. 